\title{
Military Robotics: Latest Trends and Spatial Grasp Solutions
}

\author{
Peter Simon Sapaty \\ Institute of Mathematical Machines and Systems \\ National Academy of Sciences \\ Kiev, Ukraine
}

\begin{abstract}
A review of some latest achievements in the area of military robotics is given, with main demands to management of advanced unmanned systems formulated. The developed Spatial Grasp Technology, SGT, capable of satisfying these demands will be briefed. Directly operating with physical, virtual, and executive spaces, as well as their combinations, SGT uses highlevel holistic mission scenarios that self-navigate and cover the whole systems in a super-virus mode. This brings top operations, data, decision logic, and overall command and control to the distributed resources at run time, providing flexibility, ubiquity, and capability of self-recovery in solving complex problems, especially those requiring quick reaction on unpredictable situations. Exemplary scenarios of tasking and managing robotic collectives at different conceptual levels in a special language will be presented. SGT can effectively support gradual transition to automated up to fully robotic systems under the unified command and control.
\end{abstract}

Keywords-military robots; unmanned systems; Spatial Grasp Technology; holistic scenarios; self-navigation; collective behavior; self-recovery

\section{INTRODUCTION}

Today, many military organizations take the help of military robots for risky jobs. The robots used in military are usually employed within integrated systems that include video screens, sensors, grippers, and cameras. Military robots also have different shapes and sizes according to their purposes, and they may be autonomous machines or remote-controlled devices. There is a belief that the future of modern warfare will be fought by automated weapons systems.

The U.S. Military is investing heavily in research and development towards testing and deploying increasingly automated systems. For example, the U.S. Army is looking to slim down its personnel numbers and adopt more robots over the coming years $[1,2]$. The Army is expected to shrink from 540,000 people down to 420,000 by 2019 . To keep things just as effective while reducing manpower, the Army will bring in more unmanned power, in the form of robots. The fact is that people are the major cost, and first of all their life. Also, training, feeding, and supplying them while at war is pricey, and after the soldiers leave the service, there's a lifetime of medical care to cover.

Military robots are usually associated with the following categories: ground, aerial, and maritime, with some of the latest works in all three discussed in the paper, including those oriented on collective use of robots.
Most military robots are still pretty dumb, and almost all current unmanned systems involve humans in practically every aspect of their operations. The Spatial Grasp ideology and technology described in the rest of this paper can enhance individual and collective intelligence of robotic systems, especially distributed ones. It can also pave the real way to massive use of advanced mobile robotics in human societies, military systems including and particularly.

\section{SOME LATEST DEVElopMENTS AND DEMANDS To MILITARY ROBOTICS}

\section{A. Ground Robots}

The ability of robots to save lives has secured future path for ground robotics alongside the warfighter. Ground robotics can be engaged in different missions including Explosive Ordnance Disposal (EOD), Combat Engineering, Reconnaissance, and many others. The US Army plans to refurbish 1,477 of its ground robots, which is about 60 percent of the total fleet [3]. The following may be named among the latest developments in ground robotics.

Boston Dynamics designed the LS3 "robot mules" to help soldiers carry heavy loads [4], see Fig. $1 a-c$. LS3 is a roughterrain robot designed to go anywhere Marines and Soldiers go on foot, helping carry their load. Each LS3 carries up to $400 \mathrm{lbs}$ of gear and enough fuel for a 20 -mile mission lasting 24 hours. LS3 automatically follows its leader using computer vision, so it does not need a dedicated driver. It also travels to designated locations using terrain sensing and GPS.

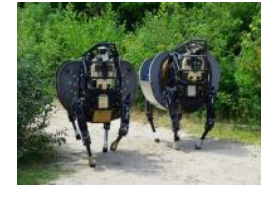

a)

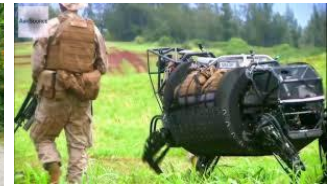

b)

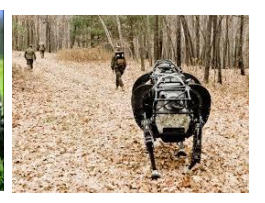

c)
Fig. 1. Boston Dynamics robot mules: a) Carrying heavy loads; b) Following soldiers; c) Moving through complex terrains

The Boston Dynamics' Cheetah robot (Fig. 2a-b) is the fastest legged robot in the World, surpassing $29 \mathrm{mph}$, a new land speed record for legged robots [5]. The Cheetah robot has an articulated back that flexes back and forth on each step, increasing its stride and running speed, much like the animal does. The current version of the Cheetah robot runs on a highspeed treadmill in the laboratory where it is powered by an 
off-board hydraulic pump and uses a boom-like device to keep it running in the center of the treadmill. a)

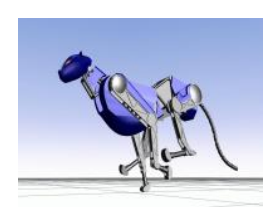

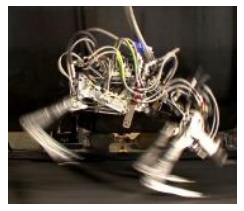

b)

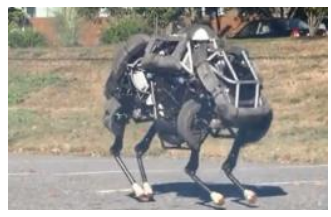

c)
Fig. 2. Boston Dynamics robots: a) The Cheetah concept; b) Cheetah on a high-speed treadmill; c) Cheetah becoming Wild Cat running untethered

The next generation Cheetah robot, WildCat, Fig. $2 c$, is designed to operate untethered. WildCat is an early model for field testing. It sports a noisy combustion onboard engine. Named the WildCat, the outdoor runner is funded by the Defense Advanced Research Projects Agency (DARPA), and is being developed for military use. With a large motor attached, WildCat isn't as fast as its $28 \mathrm{mph}$-plus cousin, being currently limited to around $16 \mathrm{mph}$ on flat terrain.

New military technology 2014 supersoldier robot has been developed [6]: all-terrain, highly mobile, and with high precision shooting (Fig. $3 a-c$ ). It is logical to assume that killer robots are already here, and the new science discoveries of 2014 may be used to create real terminators.

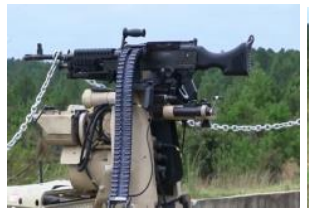

a) Ammunition

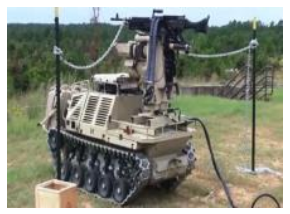

b) All terrain chassis

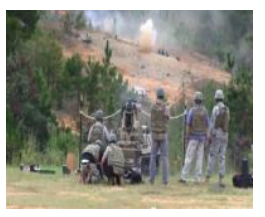

c) Field trials
Fig. 3. Supersoldier robot

\section{B. Aerial Robotics}

The US Army, Air Force, and Navy have developed a variety of robotic aircraft known as unmanned flying vehicles (UAVs). Like the ground vehicles, these robots have dual applications: they can be used for reconnaissance without endangering human pilots, and they can carry missiles and other weapons [7].

The best known armed UAVs are the semi-autonomous Predator Unmanned Combat Air Vehicles (UCAV) built by General Atomics which can be equipped with Hellfire missiles. The military services are also developing very small aircraft, sometimes called Micro Air Vehicles (MAV) capable of carrying a camera and sending images back to their base. Some newest UCAV developments are mentioned below.

The Northrop Grumman $X-47 B$ is a demonstration unmanned combat air vehicle (UCAV) designed for carrierbased operations [8], see Fig. $4 a-c$. Developed by the American defense technology company Northrop Grumman, the X-47 project began as part of DARPA's J-UCAS program, and is now part of the United States Navy's Unmanned Combat Air System Demonstration (UCAS-D) program.

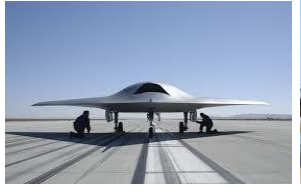

a)

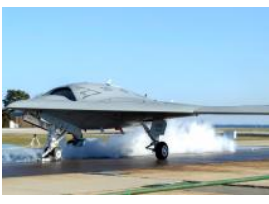

b)

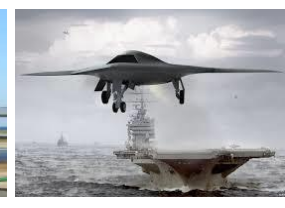

c)
Fig. 4. Northrop Grumman X-47B: a) Front view; b) Land-launched; c) Carrier-launched

The X-47B first flew in 2011, and as of 2014, it is undergoing flight and operational integration testing, having successfully performed a series of land- and carrier-based demonstrations. In August 2014, the US Navy announced that it had integrated the X-47B into carrier operations alongside manned aircraft. Northrop Grumman intends to develop the prototype X-47B into a battlefield-ready aircraft, the Unmanned Carrier-Launched Surveillance and Strike (UCLASS) system, which will enter service around 2019. X$47 \mathrm{~B}$ can stay in the air for 50 hrs, carry 2 tons of weaponry, and be refuelled in the air.

Doubling the Threat: Drones + Lasers. The research and development arm of the US Department of Defense plans to establish drone-mounted laser weapons, a scheme referred to as 'Project Endurance' in the agency's 2014 budget request [9], see Fig. 5a-c. The Pentagon edged closer to mounting missile-destroying lasers on unmanned and manned aircraft, awarding \$26 million to defense contractors to develop the technology.

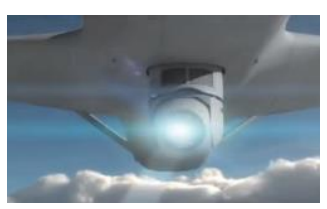

a)

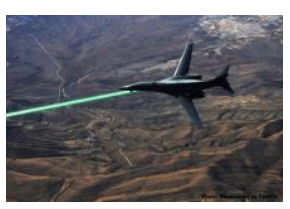

b)

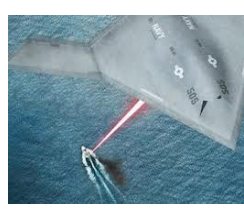

c)
Fig. 5. Drones with lasers: a) HELLADS mounted on a drone, b-c) Drone laser in operation

General Atomics is getting increasingly excited by the HELLADS - the High-Energy Liquid Laser Defense System. It is designed to shrink a flying laser into a package small enough to cram into an aircraft. This will give a potentially unlimited shooting magazine to the drone.

Hypersonic aircraft. The SR-72 [10] could fly as fast as Mach 6, will have the ability to gather intelligence, conduct surveillance and reconnaissance, and launch combat strikes at an unprecedented speed, see Fig. 6a. SR-72 could be operational by 2030. At this speed the aircraft would be so fast that adversary would have no time to react or hide.

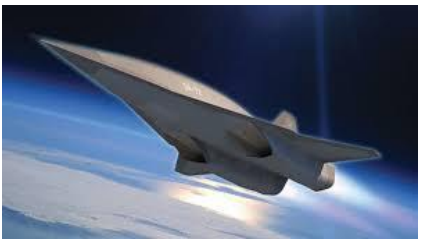

a)

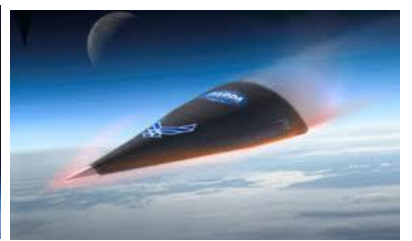

b)
Fig. 6. Hypersonic vehicles: a) SR-72 with Mach 6; b) DARPA HTV-2 with Mach 20 
DARPA rocket-launched HTV-2, 13,000 mph Hypersonic Glider [11] (see Fig. 6b), was designed to collect data on three technical challenges of hypersonic flight: aerodynamics, aerothermal effects, and guidance, navigation and control. A technology demonstration and data-gathering platform, the HTV-2's second test flight was conducted to validate current models and increase technical understanding of the hypersonic regime. The flight successfully demonstrated stable aerodynamically-controlled flight at speeds up to Mach 20.

\section{Maritime Robotics}

Sea-based robots - unmanned maritime systems, or UMSs, can be either free-swimming or tethered to a surface vessel, a submarine, or a larger robot [12], see examples in Fig. 7. Tethers simplify providing power, control, and data transmission, but limit maneuverability and range. Recently developers have built highly autonomous systems that can navigate, maneuver, and carry out surprisingly complex tasks. UMSs can operate on the ocean's surface, at or just below the surface, or entirely underwater. Operating above or near the surface simplifies the power and control, but compromises stealth. The U.S. Navy has devoted particular attention to unmanned underwater vehicles (UUSs) during the past 10-15 years. Its unmanned surface vehicles (USVs) are much less far along (Fig. 7a); the Navy has put a higher priority on using automation to reduce crew size in U.S. warships. Some latest works on UUSs follow.

Large Displacement Unmanned Undersea Vehicle (LDUUV) [13], see Fig. 7b, is to conduct missions longer than 70 days in open ocean and littoral seas, being fully autonomous, long-endurance, land-launched, with advanced sensing for littoral environments. The vehicle's manufacturing and development phase will begin in 2015 with testing planned for 2018. According to the Navy's ISR Capabilities Division, LDUUV will reach initial operating capability as a squadron by 2020 and full rate production by 2025 .
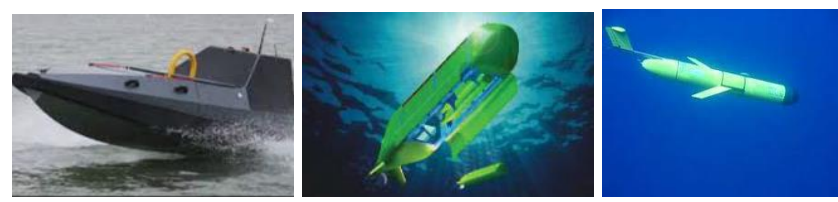

Fig. 7. a) Unmanned surface vehicle; b) Large Displacement Unmanned Undersea Vehicle, LDUUV; c) Underwater glider

Underwater gliders [14], see Fig. 7c, will not require fuel but will instead use a process called "hydraulic buoyancy," which allows the drone to move up and down and in and out of underwater currents that will help it move at a speed of about one mile per hour. Carrying a wide variety of sensors, they can be programmed to patrol for weeks at a time, surfacing to transmit their data to shore while downloading new instructions at regular intervals.

\section{Collectively Behaving Robots}

To be of real help in complex military applications, robots should be integral part of manned systems, they should also be capable of being used massively, in robotic collectives. The tests on Virginia's James River represented the first large-scale military demonstration of a swarm of autonomous boats designed to overwhelm enemies [15], see Fig. 8a. The boats operated without any direct human control: they acted as a robot boat swarm. This capability points to a future where the U.S. Navy and other militaries may deploy multiple underwater, surface, and flying robotic vehicles to defend themselves or attack a hostile force.

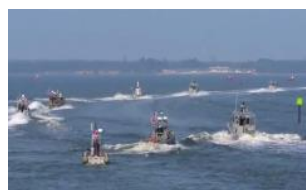

a)

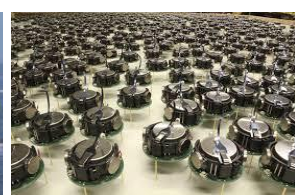

b)

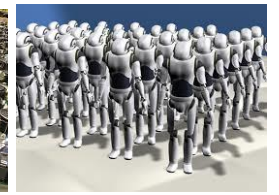

c)
Fig. 8. a) Swarm of autonomous boats; b) Harvard University multiple robots operating without central intelligence; c) Sci-fi image of future robotic armies

Harvard University scientists have devised a swarm of 1,024 tiny robots that can work together without any guiding central intelligence [16], see Fig. 8b. Like a mechanical flash mob, these robots can assemble themselves into five-pointed stars, letters of the alphabet and other complex designs. Swarm scientists are inspired by nature's team players-social insects like bees, ants and termites; schools of fish; and flocks of birds. These creatures collaborate in vast numbers to perform complicated tasks, even though no single individual is actually in charge. These results are believed to be useful for the development of advanced robotic teams even armies, (with futuristic image in Fig. 8c).

\section{E. General Demands to Military Robotic Systems}

A thorough analysis of aims and results of the development and implementation of military robots, including the ones briefed above, helps us formulate general demands with regard to their overall management and control, which may be as follows.

- Despite the diversity of sizes, shapes, and orientations, they should all be capable of operating in distributed, often large, physical spaces, thus falling into the category of distributed systems.

- Their activity is to include navigation, movement, observation, gathering data, carrying loads which may include ammunitions or weapons, and making impact on other manned on unmanned units and the environment.

- They should have certain, often high, degree of autonomy and capability of automatic decision making to be really useful in situations where human access and activity are restricted.

- They should effectively interact with manned components of the systems and operate within existing command and control infrastructures, to be integral parts of the system.

- They should be capable of effective swarming for massive use, and this swarming should be strongly controlled from outside -- from manned parts of the system or from other, higher-level, unmanned units.

- Their tasking and retasking (including that of swarms) should be flexible and convenient to humans to 
guarantee runtime reaction on changing goals and environments, especially on battlefields.

- The use of unmanned units should be safe enough to humans and systems they are engaged in.

- Their behaviour should satisfy ethical and international norms, especially in life-death situations.

\section{SPATIAL GRASP TECHNOLOGY FOR MANAGEMENT OF ROBOTIC SYSTEMS}

The developed high-level Spatial Grasp ideology and Technology, SGT, for coordination and management of large distributed systems [17] allows us to investigate, develop, simulate, and implement manned-unmanned systems in their integrity and entirety. Also gradually move to fully unmanned systems with dynamic tasking and managing individual robots and their groups, regardless of the group's size. SGT can believably satisfy most of the demands to military robotic systems formulated above.

\section{A. SGT General Issues}

SGT is based on coordinated integral, seamless, vision \& navigation \& coverage \& surveillance \& conquest of physical, virtual, or execution spaces, as shown in Fig. $9 a-b$.

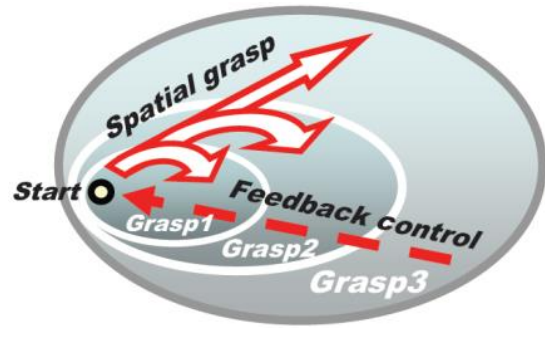

a)

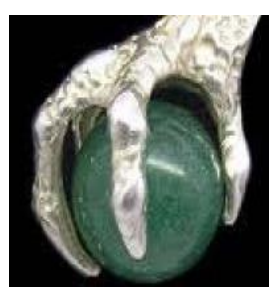

b)
Fig. 9. SGT basics: a) Controlled parallel and incremental space grasp; b) Symbolic physical analogy

It has a strong psychological and philosophical background reflecting how humans, especially top commanders, mentally plan, comprehend and control operations in complex and distributed environments. SGT pursues holistic, gestalt [18], or over-operability [19] ideas rather than traditional multi-agent philosophy [20], with multiple agents and their interactions appearing and disappearing dynamically, on the implementation level, and only if and when needed in particular places and moments of time.

SGT can be practically implemented in distributed systems by a network of universal control modules embedded into key system points (humans, robots, sensors, mobile phones, any electronic devices, etc.), which altogether, collectively, understand and interpret mission scenarios written in a special high-level Spatial Grasp Language, SGL [17], see Fig. 10.

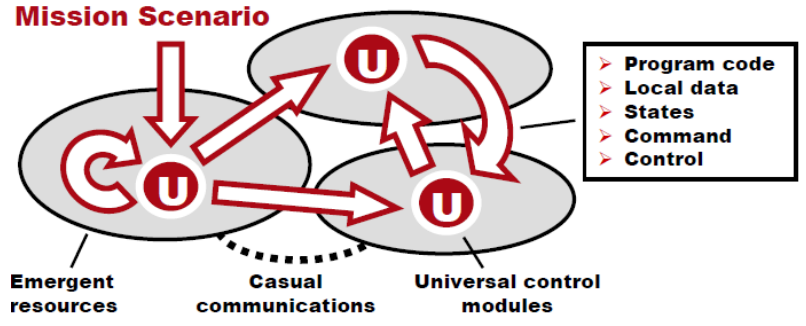

Fig. 10. Collective spatial interpretation of SGL scenarios

Capable of representing any parallel and distributed algorithms, these scenarios can start from an arbitrary node, covering at runtime the whole system or its parts needed with operations, data, and control, as shown in Fig. 11. Different scenarios can intersect in the networked space while cooperating or competing (Fig. 11).

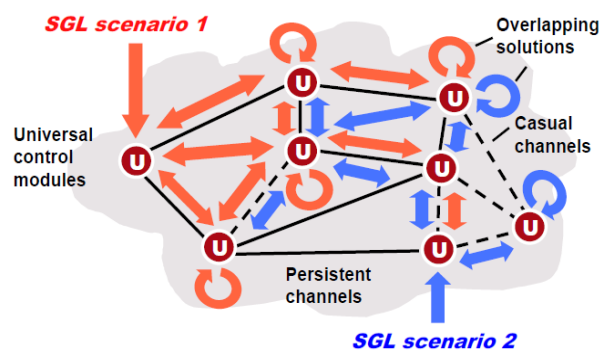

Fig. 11. Spreading scenarios intersection \& cooperation,

They can establish distributed runtime information and control infrastructures that can support distributed databases, command and control, situation awareness, autonomous decisions, also any other existing or hypothetical computational and/or control models (Fig. 12).

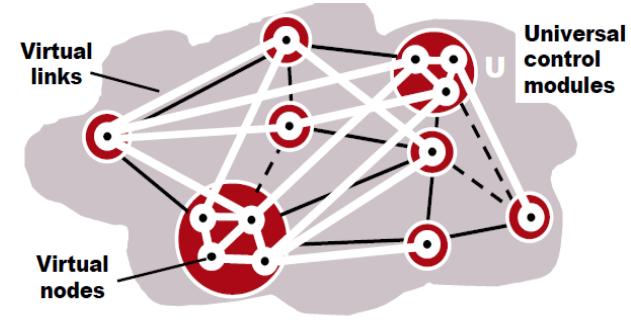

Fig. 12. Creating spatial infrastructures

\section{B. Spatial Grasp Language, SGL}

SGL allows us to directly move through, observe, and make any actions and decisions in fully distributed environments. SGL scenario develops as parallel transition between sets of progress points (or props) reflecting progressive spatial-temporal-logical stages of the scenario development, which may be associated with different physical, virtual or execution locations in distributed worlds. Any sequential or parallel, centralized or distributed, stationary or mobile algorithm operating with information and/or physical matter can be written in SGL at any levels. 
SGL directly operates with the following worlds:

- Physical World (PW), infinite and continuous, where each point can be identified and accessed by physical coordinates, with certain precision.

- Virtual World (VW), which is finite and discrete, consisting of nodes and semantic links between them.

- Executive world (EW) consisting of active doers which may be humans, robots, sensors or any intelligent machines capable of operations on matter, information, or both, i.e. on the previous two worlds.

Directly working with different worlds, SGL can provide high flexibility, convenience, and compactness in expressing complex scenarios within the same formalism. From one side, it can support high level, semantic descriptions abstracting from physical resources which can vary and be assigned at runtime, and from the other side, detailing some or all such resources, and to the full depth, if necessary.

For example, working directly with PW, like moving through and impacting it, can be free from naming physical devices which can do this (e.g. humans, robots), the latter engaged and disengaged automatically upon necessity, availability, or uselessness. Directly working with VW, like creating knowledge, operational, or C2 infrastructures, can also abstract away from physical resources (humans or computing facilities) which can be assigned or reassigned at runtime. Working directly with $\mathrm{EW}$, can bring any necessary details for execution of missions, like particular human, robotic or sensor units and their interactions and subordination. Any combination and integration of these three worlds can be possible, with direct management of the mixture in SGL too. Integration between PW and VW can be named as PVW, with other cases presented as PVW, PEW, VEW, and all three together as PVEW.

SGL has universal recursive syntactic structure shown in Fig. 13 capable of representing any parallel, distributed and spatial algorithm working with arbitrary complex data. This structure, following the spatial grasp ideology of SGT mentioned above, also allows any language obeying it to be arbitrarily extended with new operations, data and control.

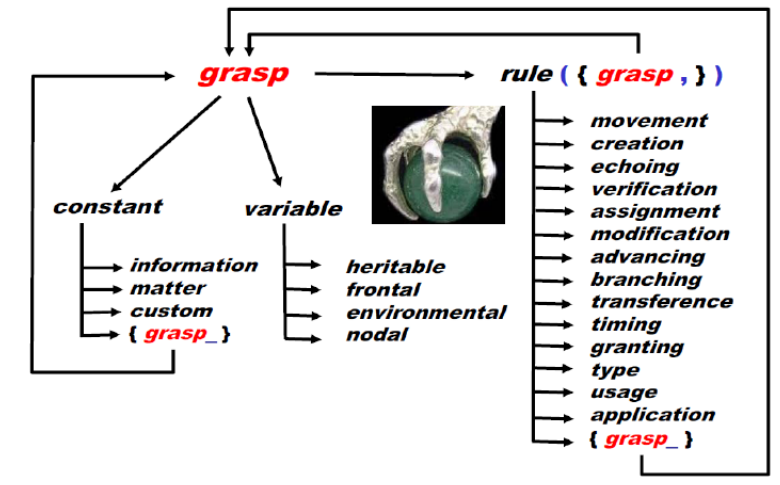

Fig. 13. Universal recursive structure of SGL
Mentioning some SGL details may be helpful for understanding the rest of this paper, as follows. The basic language construct, rule, can represent, for example, the following categories (this list being far from complete):

- Elementary arithmetic, string or logic operation.

- Hop or move in a physical, virtual, or combined space.

- Hierarchical fusion and return of local or remote data.

- Distributed control, both sequential and parallel.

- A variety of special contexts for navigation in space (influencing embraced operations and decisions).

- Type or sense of a value or its chosen usage, assisting automatic interpretation.

- Creation or removal of nodes and links in distributed knowledge infrastructures.

- Composition of other rules.

Working in fully distributed physical, virtual, executive or combined environments, SGL has different types of variables, called spatial, effectively serving multiple cooperative processes. They belong to the following four categories:

- Heritable variables - starting in a prop and serving all subsequent props which can share them in read \& write operations.

- Frontal variables - individual and exclusive prop's property (not shared with other props), being transferred between consecutive props and replicated if from a single prop a number of other props emerge thus propagating together with the evolving spatial control.

- Environmental variables - accessing different elements of the physical and virtual words when navigating them, also basic parameters of the internal world of SGL interpreter.

- Nodal variables - adding individual temporary property to VW, PW, EW or combined nodes; they can be accessed and shared by all activities currently associated with these nodes.

For simplifying and shortening complex scenarios (say, reducing nested parentheses in them), SGL programs can additionally use syntactic constructs common for traditional languages, as will be seen from the forthcoming examples of this paper, always remaining, however, within the general structure depicted in Fig. 13.

\section{Elementary Examples in SGL}

Let us consider some elementary scenarios from the mentioned three worlds (PW, VW, and EW), as shown in Fig. $14 a-f$. 


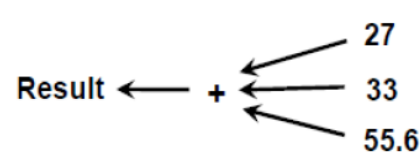

a) Assignment

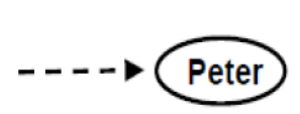

c) Virtual node creation

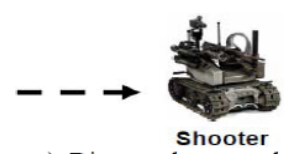

e) Direct order to a robot

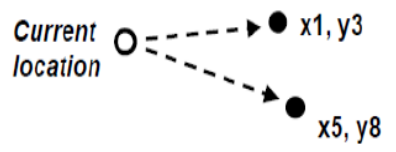

b) Physical move

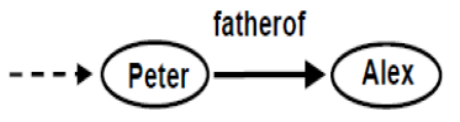

d) Semantic network extension

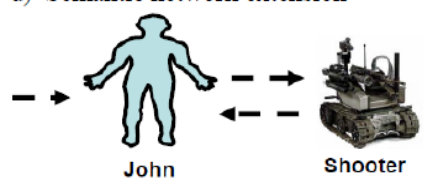

f) Direct order to a human to engage robot
Fig. 14. Some elementary scenarios for programming in SGL

They all can be expressed within the same spatial grasp ideology and unified SGL syntax, as follows.

- Assignment (Fig.14a):

assign(Result, $\operatorname{add}(27,33,55.6))$ or

Result $=27+33+55.6$

- Moves in physical space to coordinates (x1, y3), and (x5, y8) independently or in parallel (Fig. 14b):

move (location $\left(\mathrm{x}_{1}, \mathrm{y}^{3}\right)$, location $(\mathrm{x} 5, \mathrm{y} 8)$ )

- Creation of a virtual node (Fig.14c):

create ('Peter')

- Extending virtual network with a new link-node pair (Fig.14d):

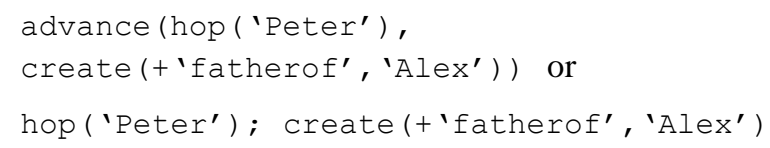

- Giving direct command to robot Shooter to fire at coordinates (x, y) (Fig. 14e):

hop (robot (Shooter)); fire(location (x,y)

- Order soldier John to fire at coordinates (x, y) by using robot Shooter and confirm robot's action in case of its success (Fig. 14f):

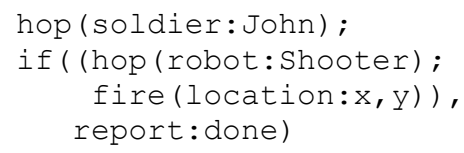

\section{SGL Interpreter Architecture}

SGL interpreter consists of specialized modules handling $\&$ sharing specific data structures, as shown in Fig. 15.

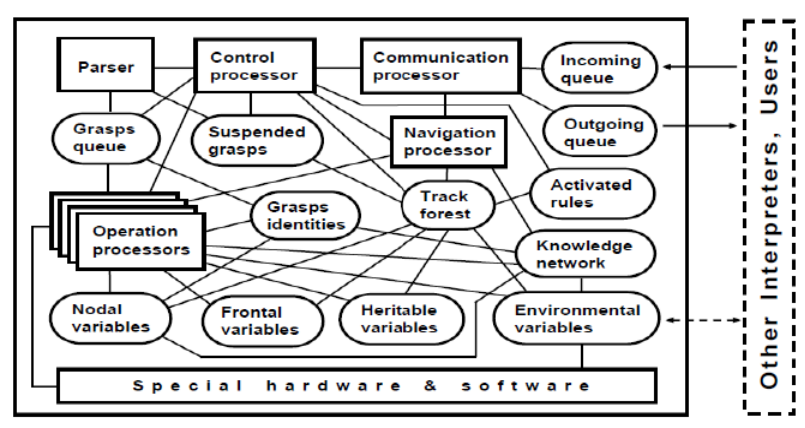

Fig. 15. SGL interpreter architecture

The network of the interpreters can be mobile and open, runtime changing the number of nodes and communication structure between them. The SGL interpreters can be concealed if to operate in hostile environments.

The dynamically networked SGL interpreters are effectively forming a sort of a universal parallel spatial machine capable of solving any problems in a fully distributed mode, without any special central resources. "Machine" rather than a computer or "brain" because it can operate with physical matter too, and can move partially or as a whole in physical environment, possibly, changing its distributed shape and the space coverage. This machine can operate simultaneously on many mission scenarios which can be injected at any time from its arbitrary nodes/interpreters.

Tracks-Based Automatic Command \& Control. The backbone and "nerve system" of the distributed interpreter is its spatial track system covering the spaces navigated and providing overall awareness, ad hoc automatic command and control of multiple distributed processes, access to and life of different types of spatial variables, as well as self-optimization and self-recovery from damages. Different stages of its operation during parallel space navigation are shown in Fig. $16 a-d$.
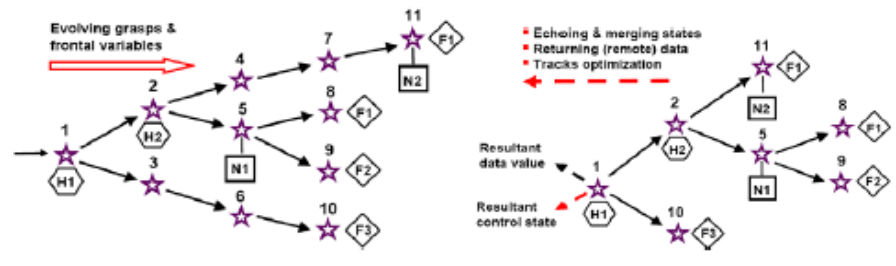

a) Forward world grasping
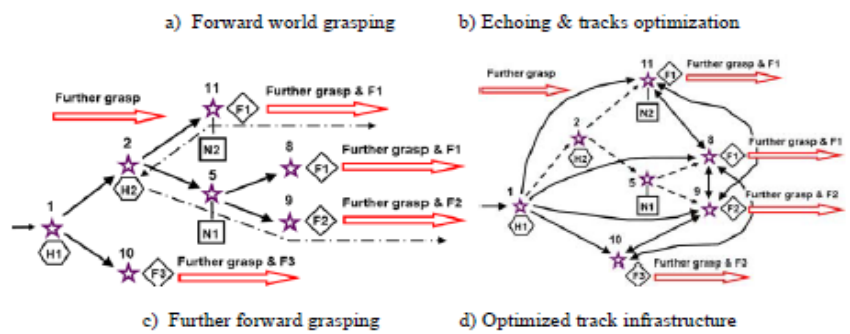

Fig. 16. The evolving track-based automatic command and control infrastructure 
The symbols in Fig. 16 have the following meanings: - nodal variables, $\diamond$ - frontal variables, $\square-$ heritable variables, $\star$ - track nodes, and $\longrightarrow$ - track links.

\section{E. Integration with Robotic Functionalities}

By embedding SGL interpreters into robotic vehicles, as in Fig. 17, we can provide any needed behavior of them, on any levels, from top semantic to detailed implementation. The technology can be used to task and control single robots as well as their arbitrary groups, with potentially unlimited number and diversity of individual robots (some hypothetic group scenarios shown in Fig. 17). For the robotic teams (or even possible future armies) it can describe and organize any collective behavior needed - from semantic task definition of just what to do in a distributed environment - to loose swarming — to a strongly controlled integral unit strictly obeying external orders. Any mixture of different behaviors within the same scenario can be guaranteed too.

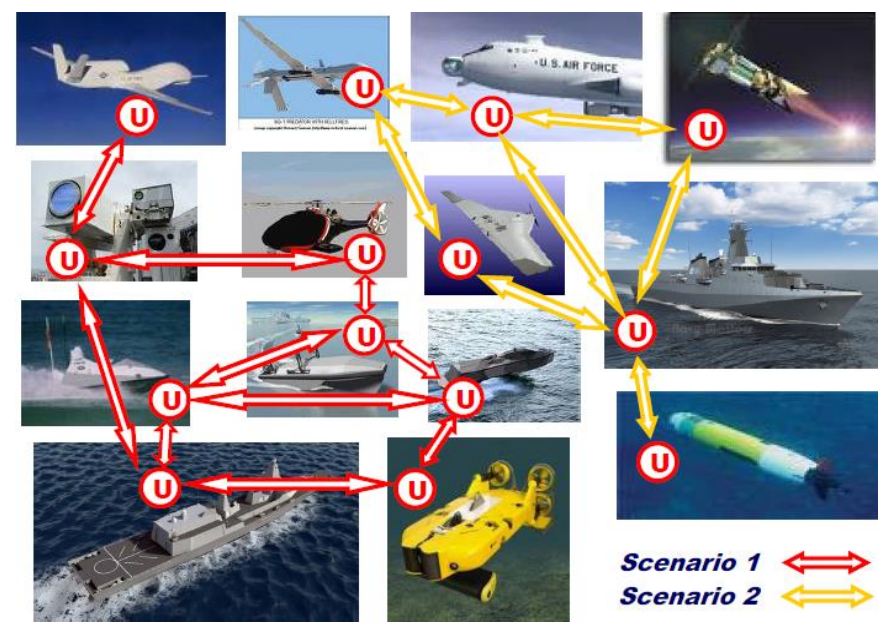

Fig. 17. Embedding SGL interpreters into robotic units and examples of collective scenarios

\section{APPLICATION OF SGT to Robotics}

\section{A. Collective Spatial Task Execution, Purely Semantic Level}

At the semantic level we can describe in SGL only what to do in a distributed space and the top decisions needed, regardless of a possible hardware or even system organization to accomplish this - these can be effectively shifted to intelligent automatic networked interpretation of the language. Let us consider the following task:

Go to physical locations of the disaster zone with coordinates:

(50.433, 30.633), (50.417, 30.490), and (50.467, 30.517).

Evaluate damage in each location and return the maximum damage value on all locations.
The corresponding SGL program will be as follows:

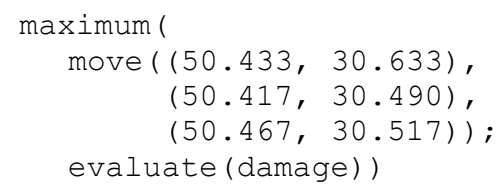

This task can be executed by different number of available mobile robots (actually from one to four, using more robots will have no much sense), and let three robots be available in the area of interest for our case, as in Fig. 18. The semantic level scenario can be initially injected into any robot (like R1), Fig. $18 a$, and then the distributed networked SGL interpreter installed in all robots automatically takes full care of the distributed task solution, with different stages depicted in Fig. $18 b-d$.

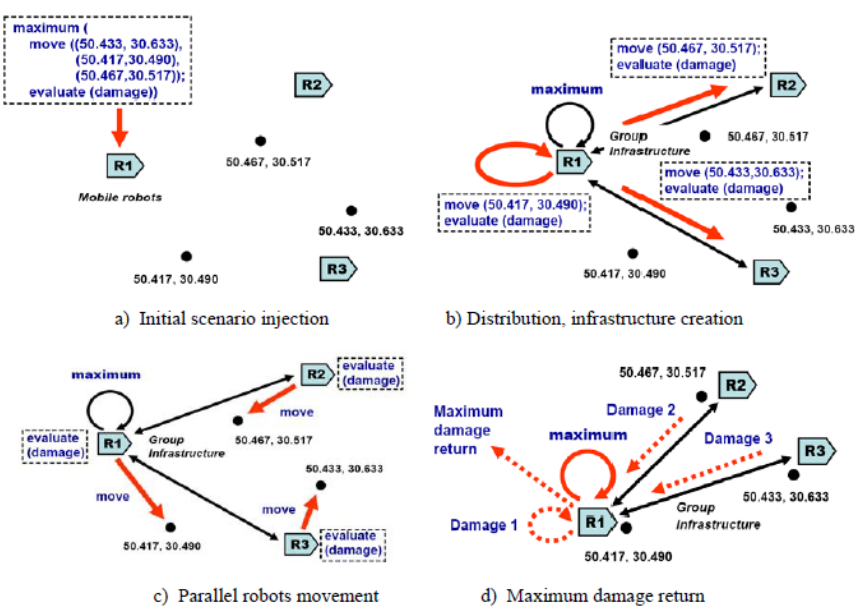

Fig. 18. Solving the task with three robots

The robots, with installed SGL interpreters and communicating with each other, are effectively forming integral distributed spatial machine that solves the problem defined purely semantically, with runtime partitioning, modifying, distributing, replicating and interlinking the emerging scenario parts automatically.

\section{B. Explicit Collective Behavior Set Up}

In contrast to the previous task defined on the level "what to do" only, different kinds of explicit behaviours can be expressed in SGL too, which, when integrated with each other, can provide very flexible, powerful, and intelligent global behaviour. Imagine that a distributed area needs to be investigated by multiple unmanned aerial vehicles that should search the space in a randomized way (preserving, however, some general direction of movement), create and update ad hoc operational infrastructure of the group (for it to follow global goal and be controlled from outside if needed), collect information on the discovered objects throughout the region covered, classifying them as targets, and organize collective reaction on the targets, as in Figure 19a- $d$. 


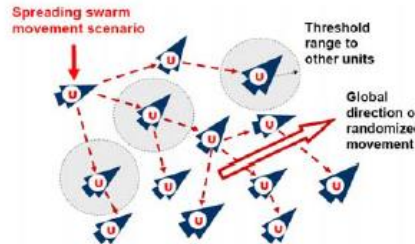

a)

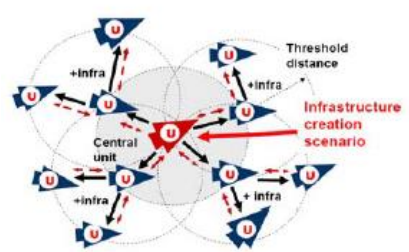

c)

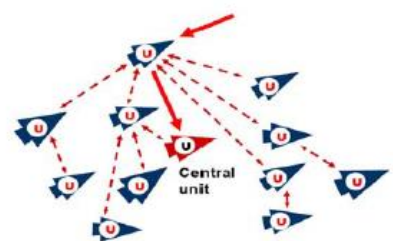

b)

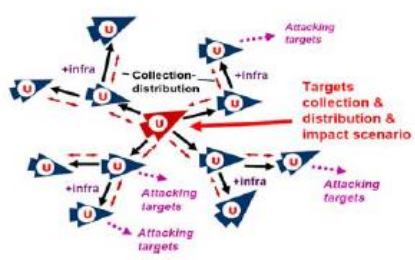

d)
Fig. 19. Different aspects of the group's behavior: a) Distributed organization of cooperative swarm movement; b) Updating \& hopping to the topological center; c) Creating \& updating of spatial runtime infrastructure starting from the updated center; d) Collecting, distributing, selecting \& attacking targets

The different stages depicted in Fig. 19 can be easily expressed in SGL and altogether integrated into the resultant holistic group scenario, as follows.

- Randomized swarm movement (starting in any node, with minimum, threshold, distance between moving nodes allowed), naming it swarm:

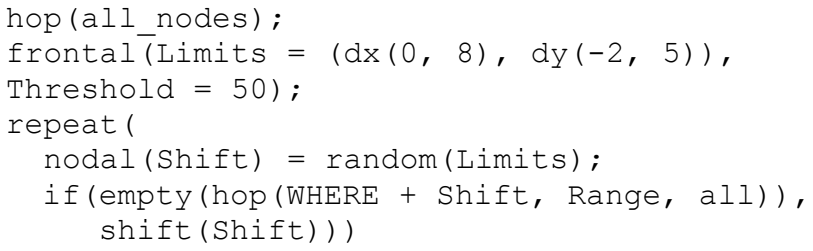

- Regular updating and subsequent hopping to topological center (as the latter may change in time due to randomized movement resulting in varying distances between nodes and also possible spatial shape of the group); starting from any node, including the current center), naming it center:

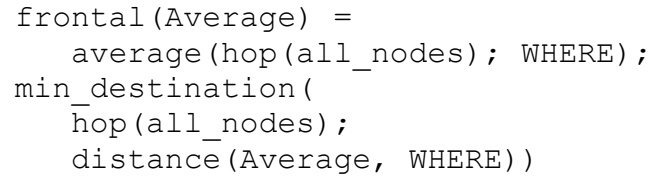

- Regular creating \& updating of spatial runtime infrastructure (starting from the updated central node, using semantic links "infra" and maximum allowed physical distance, or range, between nodes to form direct links), naming the program as infra:

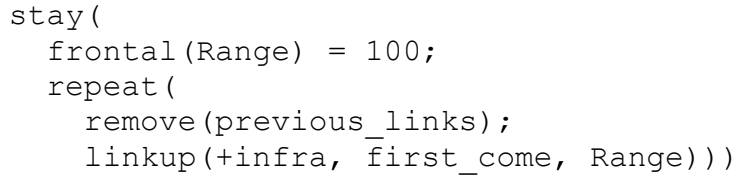

- Collecting \& selecting \& attacking targets on the whole territory controlled (starting from the updated central node and using the updated spatial infrastructure leading to all nodes, to be used repetitively until the infrastructure is updated again), let it be called targets:

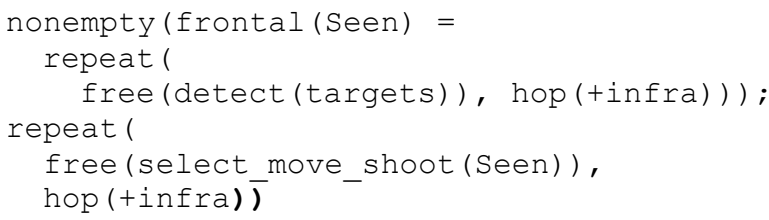

- Using these SGL scenarios for different behavioral stages, we can easily integrate them within the global one, as follows.

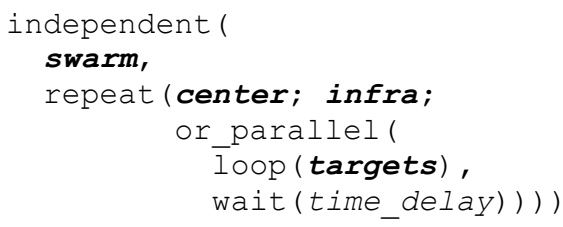

The obtained resultant scenario, which can start from any mobile unit, combines loose swarm movement in a distributed space with regular updating of topologically central unit and runtime hierarchical infrastructure between the units. The latter regularly controls observation of the distributed territory, collects data on targets and distributes them back to all units for individual selections and impact operations. The resultant scenario is setting certain time interval (time delay) for preserving status of the current central node and emanating from it infrastructure before updating them due to possible change of distances between freely moving nodes.

\section{OTHER APPLICATIONS: FORMALIZING \& AUTOMATING COMMAND AND CONTROL}

Formalization of Command Intent (CI) and Command and Control (C2) are among the most challenging problems on the way to creation of effective multinational forces, integration of simulations with live control, and transition to robotized armies. The existing specialized languages for unambiguous expression of $\mathrm{CI}$ and $\mathrm{C} 2$ (BML, C-BML, JBML, geoBML, etc.) [21] are not programming languages themselves, requiring integration with other linguistic facilities and organizational levels. Working directly with both physical and virtual worlds, SGL, being a universal programming language, allows for effective expression of any military scenarios and orders, drastically simplifying their straightforward implementation in robotized systems. SGL scenarios are much shorter and simpler than in BML or related languages, and can be created at runtime, on the fly. Typical battlefield scenario example, borrowed from [21], is shown in Fig. 20 


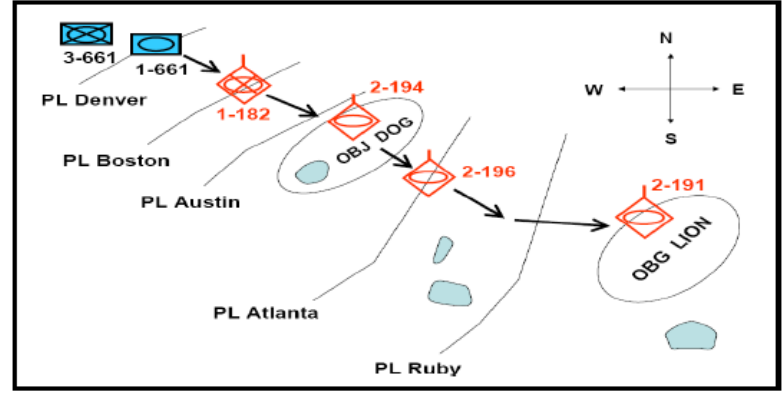

Fig. 20. Example of a battlefield scenario

The task is to be performed by two armoured squadrons BN-661 Coy1, and BN-661 Coy3, which are ordered to cooperate in coordination. The operation is divided into four time phases: from TP0 to TP1, from TP1 to TP2, from TP2 to TP3, and from TP3 to TP4, to finally secure objective LION, and on the way to it, objective DOG. Their coordinated advancement should be achieved by passing Denver, Boston, Austin, Atlanta, and Ruby lines, while fixing and destroying enemy units Red-1-182, Red-2-194, Red-2-196, and Red-2191.

This scenario can be presented in SGL as follows.

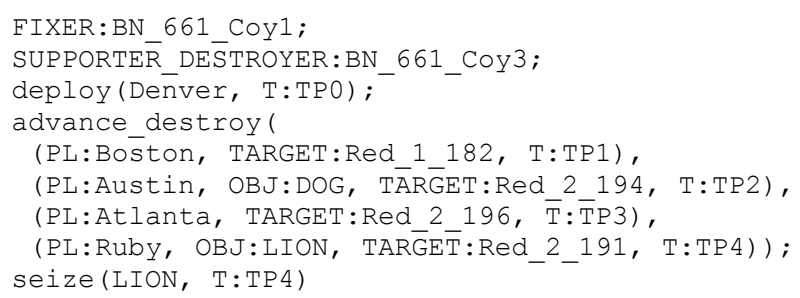

This description is much clearer, and more compact (about 10 times) than if written in BML on the level of interacting individual units, as in [21]. This simplicity may allow us redefine the whole scenario or its parts at runtime, on the fly, when the goals and environment change rapidly, also naturally engage robotic units instead of manned components. Similar to possibility of expressing different levels of organization of robotic swarms in the previous section, we may further represent this current battlefield scenario at different levels too, for example, moving upwards with its generalization, as follows:

- Not mentioning own forces, which may become clear at runtime only:

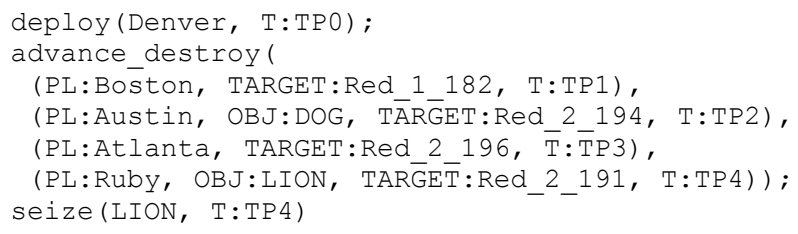

- Further up, not mentioning adversary's forces, which may not be known in advance but should be destroyed if discovered, to move ahead:

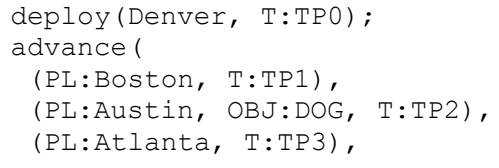

(PL:Ruby, OBJ:LION, T:TP4)) ; seize (LION, T:TP4)

- Further up, setting main stages only, with starting and final time only known:

deploy (Denver, T:TPO);

advance(PL: (Boston, Austin, Atlanta, Ruby)); seize (LION, T:TP4)

- And final goal only:

seize (LION, T:TP4)

Having the same formal language for any system levels and their any mixtures, provides us with high flexibility for organization of advanced missions, especially with limited or undefined resources and unknown environments; also possibility of potentially unlimited engagement of robotic components under the unified command and control philosophy.

\section{CONCLUSIONS}

Robots can assist humans in many areas, especially in dangerous and hazardous situations and environments. But the fate of robotics, military especially, will depend on how it conceptually and organizationally integrates with manned systems within overall management and command and control.

The developed high-level distributed control technology, SGT, based on holistic and gestalt principles can effectively support a unified transition to automated up to fully unmanned systems with massive use of advanced robotics. The practical benefits may be diverse and numerous. One of them, for example, may be effective management of advanced robotic collectives, regardless of their size and spatial distribution, by a single human operator only, due to high level of their internal self-organization and integral responsiveness provided by SGT. More on the SGT philosophy and history, details of SGL with its networked implementation, and the researched applications, some of which have been mentioned throughout this paper, can be found elsewhere [22-28].

\section{REFERENCES}

[1] "U.S. Army Considers Replacing Thousands of Soldiers with Robots", U.S.S. Enterprise, IEEE Starship U.S.S Enterprise Section, 2015, http://sites.ieee.org/uss-enterprise/u-s-army-considers-replacingthousands-of-soldiers-with-robots/.

[2] E. Ackerman, "U.S. Army Considers Replacing Thousands of Soldiers with Robots”, IEEE Spectrum, 22 Jan 2014, http://spectrum.ieee.org/automaton/robotics/military-robots/armyconsiders-replacing-thousands-of-soldiers-with-robots.

[3] "US Army Works Toward Single Ground Robot", Defense News, Nov. 15, 2014, http://archive.defensenews.com/article/20141115/DEFREG02/31115003 3/US-Army-Works-Toward-Single-Ground-Robot.

[4] "LS3 - Legged Squad Support Systems", Boston Dynamics, http://www.bostondynamics.com/robot_ls3.html.

[5] "CHEETAH - Fastest Legged Robot", Boston Dynamics, 2013, http://www.bostondynamics.com/robot_cheetah.html.

[6] W. Rodriguez, "New Military Technology 2014 Supersoldier Robot Developed", Latest New Technology Gadgets, Sept. 28, 2014, http://latestnewtechnologygadgets.com/wp/new-military-technology2014-supersoldier-robot-developed/.

[7] P. Lin, G. Bekey, K. Abney, "Autonomous Military Robotics: Risk, Ethics, and Design". US Department of Navy, Office of Naval Research 
December

20,

2008.http://www.unog.ch/80256EDD006B8954/(httpAssets)/A70E329D

E7B5C6BCC1257CC20041E226/\$file/Autonomous+Military+Robotics

+Risk,+Ethics,+and+Design_lin+bekey+abney.pdf.

[8] "X-47BUCAS, Capabilities", Northrop Grumman, 2015, http://www.northropgrumman.com/Capabilities/X47BUCAS/Pages/defa ult.aspx.

[9] A. McDuffee, "DARPA Plans to Arm Drones With Missile-Blasting Lasers", WIRED, 11.01.13, http://www.wired.com/2013/11/dronelasers/.

[10] "Meet the SR-72", Lockheed Martin, 2013, http://www.lockheedmartin.com/us/news/features/2013/sr-72.html.

[11] Engineering Review Board Concludes Review of HTV-2 Second Test Flight, DARPA, April 20, 2012, http://www.darpa.mil/newsevents/releases/2012/04/20.aspx.

[12] B. Berkowitz, "Sea Power in the Robotic Age", ISSUES in Science and Technology, 2015, http://issues.org/30-2/bruce-2/.

[13] "Large Displacement Unmanned Underwater Vehicle Innovative Naval Prototype (LDUUV INP)", in Naval Drones, http://www.navaldrones.com/LDUUV-INP.html.

[14] Wood, Stephen, “Autonomous Underwater Gliders", Florida Institute of Technology, http://my.fit.edu/ swood/26_Wood_first.pdf.

[15] J. Hsu, "U.S. Navy Tests Robot Boat Swarm to Overwhelm Enemies", IEEE Spectrum, $\quad 5 \quad$ Oct 2014. http://spectrum.ieee.org/automaton/robotics/military-robots/us-navyrobot-boat-swarm.

[16] R. L. Hotz, "Harvard Scientists Devise Robot Swarm That Can Work Together". The Wall Street Journal. Aug. 15, 2014. http://www.wsj.com/articles/harvard-scientists-devise-robot-swarm-thatcan-work-together-1408039261.

[17] P. Sapaty, “The World as an Integral Distributed Brain under Spatial Grasp Paradigm", book chapter in Intelligent Systems for Science and
Information, $\quad$ Springer, $\quad$ Feb $\quad 4, \quad 2014$ http://link.springer.com/chapter/10.1007/978-3-319-04702-7_4.

[18] M. Wertheimer, Gestalt theory. Erlangen, Berlin. 1924.

[19] P.S. Sapaty, "Over-Operability in Distributed Simulation and Control", The MSIAC's M\&S Journal Online, Winter 2002 Issue, Volume 4, No. 2, Alexandria, VA, USA.

[20] M. Minsky, The Society of Mind, Simon \& Schuster, 1988.

[21] U. Schade, M. R. Hieb, M. Frey, K. Rein, "Command and Control Lexical Grammar (C2LG) Specification”, FKIE Technical Report ITF/2010/02, July 2010.

[22] P.S. Sapaty, "Unified Transition to Cooperative Unmanned Systems under Spatial Grasp Paradigm”, International journal Transactions on Networks and Communications (TNC), Vol.2, Issue 2, Apr 2014 http://scholarpublishing.org/index.php/TNC.

[23] P.S. Sapaty, "Distributed Human Terrain Operations for Solving National and International Problems", International Relations and Diplomacy, Vol. 2, No. 9, September 2014. http://www.davidpublishing.com/journals_info.asp?jId=2094.

[24] P.S. Sapaty, "From Manned to Smart Unmanned Systems: A Unified Transition", SMi's Military Robotics, Holiday Inn Regents Park London, 21-22 May 2014. http://www.smionline.co.uk/defence/archive/5-2014/conference/military-robotics.

[25] P.S. Sapaty, "Integration of ISR with Advanced Command and Control for Critical Mission Applications", SMi's ISR conference, Holiday Inn Regents Park, London, 7-8 April 2014. http://www.smionline.co.uk/defence/archive/4-2014/conference/isr.

[26] P.S. Sapaty, Ruling distributed dynamic worlds. John Wiley \& Sons, New York, 2005.

[27] P.S. Sapaty, Mobile processing in distributed and open environments. John Wiley \& Sons, New York, 1999.

[28] P.S. Sapaty, A distributed processing system. European Patent No. 0389655, Publ. 10.11.93, European Patent Office, 1993. 